\title{
Analysis of Gut Microbiome and Diet Modification in Patients with Crohn's Disease
}

\author{
Sumathi Sankaran Walters ${ }^{1 *}$, Antonio Quiros ${ }^{2,3}$, Matthew Rolston ${ }^{1}$, Irina Grishina ${ }^{1}$, Jay Li $^{1}$, \\ Anne Fenton ${ }^{1}$, Todd Z. DeSantis ${ }^{4}$, Anne Thai ${ }^{2}$, Gary L Andersen ${ }^{5}$, Peggy Papathakis ${ }^{6}$, Raquel \\ Nieves $^{7}$, Thomas Prindiville ${ }^{2}$ and Satya Dandekar ${ }^{1}$ \\ ${ }^{1}$ Department of Medical Microbiology and Immunology, University of CA Davis, USA \\ ${ }^{2}$ Department of Internal Medicine, University of CA Davis, Sacramento, USA \\ ${ }^{3}$ Division of Pediatric Gastroenterology MUSC Children's Hospital, Charleston, SC, \\ ${ }^{4}$ Department of Bioinformatics, Second Genome, San Bruno, CA 94066. \\ ${ }^{5}$ Ecology Department, Lawrence Berkeley National Laboratory, Berkeley, CA 94720 \\ ${ }^{6}$ Department of Nutrition, California Polytechnic State University, San Luis Obispo, CA, USA \\ ${ }^{7}$ Department of Pediatrics, David Grant Medical Center, Travis Air Force Base, California, 94535
}

Received: February 24, 2014; Accepted: June 24, 2014; Published: June 27, 2014

*Corresponding author: Sumathi Sankaran Walters, Department of Medical Microbiology and Immunology, University of CA Davis, 5605A GBSF, One Shields Ave, Davis, CA 95616, USA, Ph: 530-754-7242; Fax: 530-754-7240, E-Mail: ssankara@ucdavis.edu

\begin{abstract}
Objective: The human intestine harbors trillions of commensal microbes that live in homeostasis with the host immune system. Changes in the composition and complexity of gut microbial communities are seen in inflammatory bowel disease (IBD), indicating disruption in host-microbe interactions. Multiple factors including diet and inflammatory conditions alter the microbial complexity. The goal of this study was to develop an optimized methodology for fecal sample processing and to detect changes in the gut microbiota of patients with Crohn's disease receiving specialized diets.
\end{abstract}

Design: Fecal samples were obtained from patients with Crohn's disease in a pilot diet crossover trial comparing the effects of a specific carbohydrate diet (SCD) versus a low residue diet (LRD) on the composition and complexity of the gut microbiota and resolution of IBD symptoms. The gut microbiota composition was assessed using a high-density DNA microarray PhyloChip.

Results: DNA extraction from fecal samples using a column based method provided consistent results. The complexity of the gut microbiome was lower in IBD patients compared to healthy controls. An increased abundance of Bacteroides fragilis (B. fragilis) was observed in fecal samples from IBD positive patients. The temporal response of gut microbiome to the SCD resulted in an increased microbial diversity while the LRD diet was associated with reduced diversity of the microbial communities.

Conclusion: Changes in the composition and complexity of the gut microbiome were identified in response to specialized carbohydrate diet. The SCD was associated with restructuring of the gut microbial communities.

Keywords: IBD; Crohn's Disease; Fecal microbiome; Diet Modification; PhyloChip

\section{Summary}

What is already known about this subject?

- The interplay between the gut microbiota and mucosal health is of great importance especially in Inflammatory

\section{Bowel Disease}

- The gut microbial balance can be altered by modifying the diet.

- Taken together, the goal of this study was to follow changes in the gut microbiota by longitudinally analyzing the fecal microbiome in patients with IBD.

What are the new findings?

- The study has identified restricted microbial diversity associated with IBD.

- Our study has demonstrated, for the first time, an enrichment of microbial diversity following the use of the specific carbohydrate diet.

How might it impact on clinical practice in the foreseeable future?

- Diet modification will be an important means to reduce symptoms and improve mucosal healing

- Additional microbial modification using Probiotics could be beneficial.

- Expansion of this study model can provide a definitive means to improve microbial diversity and mucosal health in IBD patients.

\section{Introduction}

Human intestine harbors trillions of diverse communities of commensal bacteria that are beneficial for the human health [1-4]. It has been estimated that over 100 trillion microbes belonging to over 500 species co-exist in the human colon [5]. Changes in the composition of intestinal microbiota have been observed in disease involving infectious and non-infectious 
etiologies [6-10]. Diet is known to cause changes in fecal Microbiota [11,12]. Three predominant enterotypes have been shown to be present in the human gut microbiome: Bacteroides, Prevotella and Ruminococcus [13]. Several studies suggest that each individual harbors his or her own distinctive pattern of intestinal microflora composition that is not necessarily distinct but is a gradient microbial community [14]. This pattern tends to remain constant across time with the exception of possible age and Body Mass Index (BMI) related changes [15-17]. IBD and gastrointestinal cancers are thought to affect the microflora composition and recent data supports such association [9,1821]. Human feces provide a complex microbial niche and are reflective of the microbiota that is present in the large intestine [22-25]. Thus, analysis of the bacterial communities in human feces is widely utilized to determine the changes in infectious or non-infectious diseases.

Culture-independent molecular methodologies have facilitated more accurate and rapid detection of changes in microbial communities during various disease states [26,27]. The detection of a broad-range of commensal bacteria is based on classifying heterogeneous $16 \mathrm{~S}$ genes amplified by polymerase chain reaction (PCR) using primers with broad specificity [28-30]. Prior to 2010 the general method of sampling and categorizing DNA from complex samples has been to clone or otherwise segregate individual amplicons, then sequence a subset of them [28-30]. However, the number of sequences required to adequately quantify the population levels of each taxa in a sample is unwieldy. Additionally, newer high throughput sequencing technology has been used to study bacterial populations using the $16 \mathrm{~S}$ sequences. While this technology is readily available the complex analytical tools and expertise necessary to analyze the data is not as readily available. Recent development of a high density microarray consisting of probes complementing $16 \mathrm{~S}$ genes has enabled researchers to perform profiling of the microbes in a given sample for monitoring changes in the bacterial communities in a simple and time efficient manner [31]. These microarrays have been used in monitoring microbes in diverse environments and in disease and are able to provide a fingerprint of characterized sequences [32-34]. Specific microbiome signature changes have been reported in inflammatory bowel disease, obesity and infectious diseases and are considered for the diagnosis and monitoring of these disorders. This provides an opportunity to develop more novel biomarkers for monitoring disease progression or therapeutic outcomes.

The use of probiotics, dietary patterns and intake of various nutrients can change profiles of fecal microflora [12, 35-37]. Specific diets have been implicated to play a role in Crohn's disease. Thus, efforts were made to utilize specialized diets, such as the Specific Carbohydrate Diet ${ }^{\mathrm{TM}}$ (SCD) and the Low Residue Diet (LRD), for restoring the gut microbiome and to improve intestinal health [38-41]. The SCD restricts the use of complex carbohydrates and has been advocated in the symptomatic management of celiac disease for many years since 1955 [42-45]. The LRD is a low fiber diet and serves to prolong intestinal transit time. This has been suggested to be beneficial in alleviating
Crohn's disease symptoms with varying results [46]. Further investigations are needed to gain insights into the complexity and dynamics of the human microbiota in the context of various diets for managing gut inflammation.

In this study, we investigated several methods of fecal sample processing for their utility in providing the best representation of the fecal microbiome diversity. The aim of this study was to demonstrate changes in the complexity of the microbiome in the longitudinal fecal samples following diet modification using culture independent high-density microarrays, the $16 \mathrm{~S}$ PhyloChip. We examined the impact of IBD as well as the SCD or LRD diet modification on the restructuring of the gut microbiota.

\section{Methods}

\section{Study subjects and experimental design}

This study was designed in part to investigate the effect of DNA extraction methods on the ability to detect changes in the complexity of fecal microbiome of healthy controls and patients with Crohn's disease and to investigate the effects that diet has on restoring the gut microbial complexity in patients with Crohn's disease. The SCD and LRD were selected to investigate their effects on the alleviation of the clinical symptoms of Crohn's disease. Previous anecdotal reports suggested a wide use of LRD by patients and a potential of enhanced relief from clinical symptoms of Crohn's disease with the use of SCD [47-49]. Participants were enrolled in the study at the pediatric and adult IBD clinics at UC Davis Medical Center (Sacramento, CA) as per IRB approved protocols.

Eight participants, aged 16-50 years (Table 1) who met the inclusion criteria were enrolled in this study. All participants were Caucasian. Two participants were healthy controls while six participants had a diagnosis of Crohn's disease (CD). Inclusion criteria for the CD cohort included: 1) confirmed diagnosis of CD, 2) in clinical remission 3) no Probiotics use and 4) willing to sign consent form for enrollment into the trial. Exclusion criteria included: 1) failure to meet any of the inclusion criteria, 2) poor compliance with the diet during the study phase, 3) failure to submit stool samples as indicated at each phase of the study, and 4) need for antibiotic use during the study. Subjects were randomized to either diet A (LRD) or diet B (SCD) and an education booklet was provided to them describing the research diet and sample menus. Stool samples were collected on day 1 and 30 (Figure 1). A twenty four-hour dietary recall was performed by the trained dietitians over the phone. After their initial 30 day period, patients went on a "washout" phase for 30 days. During this period the participants resumed their normal pre-study diet. After washout period, patients returned to clinic and the second 30-day trial period began. Stool samples were collected again at the beginning and after 30 days on the trial diet. Standard IBD questionnaires (IBDQ) were collected from all subjects at the beginning and end of the study to access clinical outcome. Loss of compliance or worsening of the clinical disease state automatically excluded them from continuing the study.

All patients were blind to the specific names and any 
Table 1: Participant Characteristics.

\begin{tabular}{|c|c|c|c|c|c|c|}
\hline Participant ID & Gender & Age & Duration of Disease(Yrs) & Diagnosis/Location & Prior Surgery & \\
\hline IBD1 & F & 44 & 2 & CD ileocolon & none & none \\
\hline IBD2 & F & 30 & 9 & CD ileum & $6 M$ MPemicade & Small Bowel Resection \\
\hline IBD3 & F & 16 & 5 & CD ileocolon & $6 \mathrm{MP} /$ Remicade & none \\
\hline IBD4 & F & 38 & 22 & CD ileocolon & $6 \mathrm{MP} /$ Remicade & none \\
\hline IBD5 & F & 18 & 8 & CD Small Bowel & $6 \mathrm{MP} /$ Remicade & Small Bowel Resection \\
\hline
\end{tabular}

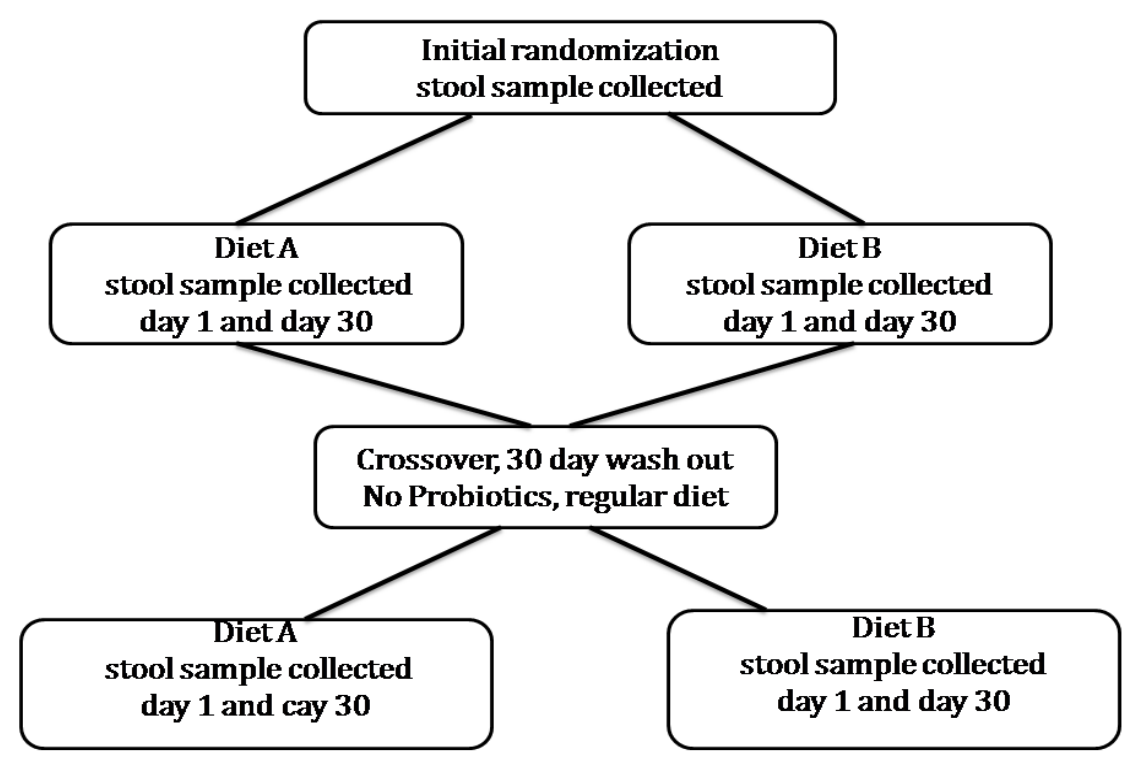

Figure 1: Schematic of the study design to analyze the effects of Diet A and Diet B on fecal microbiome: Participants were randomized in one of two arms of the study, Diet A or Diet B for 30 days, followed by a 30 days washout period and then switch to the other diet A or $\mathrm{B}$ for 30 days. Fecal samples were obtained at the start and end of each diet.

commercial affiliations of the research diet they were on and were not aware of the specifics regarding the diet they were on. The IBD care team was also blinded to the diet assignments. Research dieticians performed randomization and subject education for all pediatric and adult subjects. Education packets on both SCD and LRD diets were carefully developed. SCD dietary information was carefully reviewed to follow dietary guidelines as described by E. Gottschall. Sample menus were developed from her book "Breaking the vicious cycle." Ms. Gottschall and her estate approved use of the material prior to starting this study. LRD instructions were crafted to mimic and display no inferiority to the SCD booklet $[50,51]$. This was done to avoid induction of bias into our subject population. Two healthy participants with no gastrointestinal symptoms or other chronic illnesses were also enrolled to provide a baseline control fecal sample [52,53].

\section{DNA extraction from fecal samples}

An initial stool sample, IBD3-01, was used to test for efficacy and reliability of the bacterial DNA extraction method. Two DNA extraction methods, P1 (Phenol Chloroform: P) and Q1 (Qiagen Stool Kit: Q), were used and compared (Figure 2). Two separate $250 \mu \mathrm{L}$ stool samples were used for the Q1 test, whereas for P1
$500 \mu \mathrm{L}$ of sample was digested overnight, and then split into two $250 \mu \mathrm{L}$ samples for P1 extraction. The four resulting DNA extracts were diluted to test for optimal PCR template concentration. $4 \mathrm{ng}$, $20 \mathrm{ng}$, and $100 \mathrm{ng}$ of each of the four templates were individually amplified by PCR for bacterial 16S rRNA gene (16S) and for archaeal 16S. Each dilution was amplified independently eight times on a temperature gradient of $48^{\circ} \mathrm{C}$ to $58^{\circ} \mathrm{C}$. PCR products were visualized on $1 \%$ agarose gels containing ethidium bromide. 20 ng of DNA template consistently amplified well, and the amplifications from $48^{\circ} \mathrm{C}-58^{\circ} \mathrm{C}$ showed larger bands. All subsequent PCRs were done with 20 ng total DNA templates on a gradient of $48^{\circ} \mathrm{C}$ to $58^{\circ} \mathrm{C}$ to decrease the likelihood of missing sensitive bacterial populations [54,55]. Variations to the starting materials included: P2: $250 \mathrm{mg}$ of feces instead of $250 \mathrm{uL}$. P3: Same as P1 but using $250 \mathrm{uL}$ of feces in Trizol. Q1: $250 \mathrm{uL}$ of frozen stool sample. Extract using Qia Amp DNA Stool Miniprep Kit as described by manufacturers. Elute in 50 uL Buffer AE. Q2: Same as Q1 but use $250 \mathrm{mg}$ of frozen feces. Q3: Same as Q1 but use $250 \mathrm{uL}$ of feces in Trizol.

The IBD4-02 DNA was extracted with both the P2 and Q2 methods to test extraction comparability again while standardizing for mass. The sample was then extracted using 


\begin{tabular}{|c|c|c|c|}
\hline IBD3 01 & $\begin{array}{l}\text { Q2 ( } 250 \text { ug Fecal) } \\
\text { Q1 (250ul Fecal) } \\
\text { P1 (250 ul Fecal) } \\
\text { P2 (250 ug Fecal) }\end{array}$ & $\begin{array}{l}\text { Q2 vs } Q 1 \\
\text { Q2 vs } P 2\end{array}$ & $\begin{array}{l}P 1 \text { vs } P 2 \text { effect of st } \\
Q 1 \text { vs } P 1 \text { effect of } C\end{array}$ \\
\hline IBD4 02 & 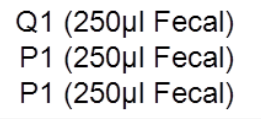 & $\begin{array}{l}P 1 \text { vs } P 1 \\
\text { Q1 vs } P 1\end{array}$ & $\begin{array}{l}\text { - reproducibility } \\
\text { - effect of extraction }\end{array}$ \\
\hline
\end{tabular}

Figure 2: Schematic of the extraction controls: IBD301 and IBD402 are individual fecal samples. P indicates extraction using the phenol-chloroform method. Q indicates extraction using the Qiagen Fecal DNA extraction method. The analysis strategy is also outlined.

the P3 and Q3 methods to test whether extracting from stool samples in Trizol (Invitrogen) would give different results than performing extraction from the original fecal sample (Figure 2).

\section{S sequence amplification by polymerase chain reaction assay and hybridization}

Bacterial DNA amplification was done on a Master cycler gradient thermocycler (Eppendorf). The $50 \mu \mathrm{L}$ reaction mixture contained $21.75 \mu \mathrm{L}$ of sterile water, $5 \mu \mathrm{L}$ of 10X PCR Buffer (Takara), $0.25 \mu \mathrm{L}$ of $5 \mathrm{U} / \mu \mathrm{L}$ ExTaq (Takara), $4 \mu \mathrm{L}$ of $2.5 \mathrm{mM}$ $\mathrm{dNTP}, 5 \mu \mathrm{L}$ of $10 \mathrm{mg} / \mathrm{mL}$ BSA (New England Biolabs), $5 \mu \mathrm{L}$ each of $3 \mu \mathrm{M}$ forward and reverse primers, and $4 \mu \mathrm{L}$ of template DNA. Bacterial primers were $27^{\circ} \mathrm{F}$ ( $5^{\prime}$ - agagtttgatcctggctcag- $\left.3^{\prime}\right)$ and 1492R (5'- ggttaccttgttacgactt-3'). Archaeal primers were $4 \mathrm{Fa}$ (5'-tccggttgatcctgccrg-3') and 1492R (5'- ggttaccttgttacgactt-3'). PCR products were hybridized to the Phylochips as previously described [56]. Briefly, the hybridization method is similar to the standard Affymetrixhybridization protocol.Control oligo used was a DOE 213 primer (5' biotin-7 TCCTGAACGGTAGCATCTTGACGAC $\left.3^{\prime}\right)$, and a custom spike-in control of known bacterial DNAs was added to the fragmentation mix during DNA fragmentation.

\section{Data analysis}

Cell files were analyzed with PhyloTrac(Institute for Genome Sciences, Baltimore, USA) and dCHIP(Harvard School of Public Health, Boston, USA). PhyloTrac implements background subtraction, normalization and probe scoring algorithms as previously described [57]. DChip was used to do a basic comparison of samples as previously described [58, 59]. Only samples with a pf score $>0.9$ were analyzed.

\section{Results}

\section{Participants}

Eleven subjects were approached about study participation. Six subjects met inclusion criteria and were enrolled in the trial. All subjects were females, between 16 to 48 years of age, and on Imuran for remission maintenance (Table 1). Two patients withdrew from the study due to inability to comply with dietary recommendations of which 1 patient provided a pre diet modification sample. In total, 17 fecal samples were obtained from 5patients enrolled in the trial, of which 16 samples were utilized for analysis of dietary associated changes in the fecal microbiome and 5 samples for comparison of IBD vs controls. Compliance with the diets, based on weekly phone contacts, was approximately $80 \%$. All subjects submitted fecal samples at four time points, at the beginning and end of each research diet. A four-week washout phase was kept for all subjects (Figure 2). For the duration of the study period, Crohn's disease medications and dosages were unchanged.

\section{Fecal sample processing for DNA isolation impacts microbiome profiles}

The bacterial microbiome profiles depended to a great extent on the methodology used to extract bacterial DNA from the fecal samples. This is in agreement with previous findings using colonic mucosa [54]. The 7 fecal samples used to compare the methodology were analyzed using hierarchical clustering. All samples that were derived from Qiagen stool DNA extraction method clustered together (IBD3-01-Q1, IBD3-01-Q2 and IBD402-01-Q1) (Figure 3). Because duplicate extractions using each method were done, it was possible to test consistency within methods. The duplicates had very similar profiles (P1 duplicates average expression ratio: 0.94; Q1 duplicates average expression ratio: 1.08), indicating a high level of reliability. The P1 extractions generally had higher fluorescence (P1:Q1 average ratio: 1.60) for each probe (bacterial subspecies). P2 and Q2 were used on the IBD4-02 sample to test whether the results would apply across samples. This time, the Q2 had higher fluorescence than P2 (P1:Q1 average ratio: 1.81) Again, P2 and Q2 gave similar bacterial population profiles, but P2 expressed much lower levels of Bacteroidetes spp. The results from the P3 extraction of IBD402 were similar to P2 though with higher fluorescence (P3:P2 average expression ratio: 1.45). Q3 did not produce enough DNA to amplify (Data not shown).While all samples from IBD301 clustered together; samples using the Qiagen extraction method were more closely clustered. Furthermore samples from the same participant extracted using the same methods were most closely related irrespective of the amount of starting material.

\section{Differential detection of Bacteroidetes based on bacterial DNA extraction method}

The phenol based extractions had a much lower representation of Bacteroidetes spp. population than the Qiagen based extraction (P1:Q1 average Bacteroidetes spp. ratio: 0.60) (Figure 4A). Hierarchical clustering of the Bacteroides family showed the highest representation in the three samples that were processed using the column extraction method (Qiagen), 66 species were represented on the phylochip. From 54 to $62 \%$ of 


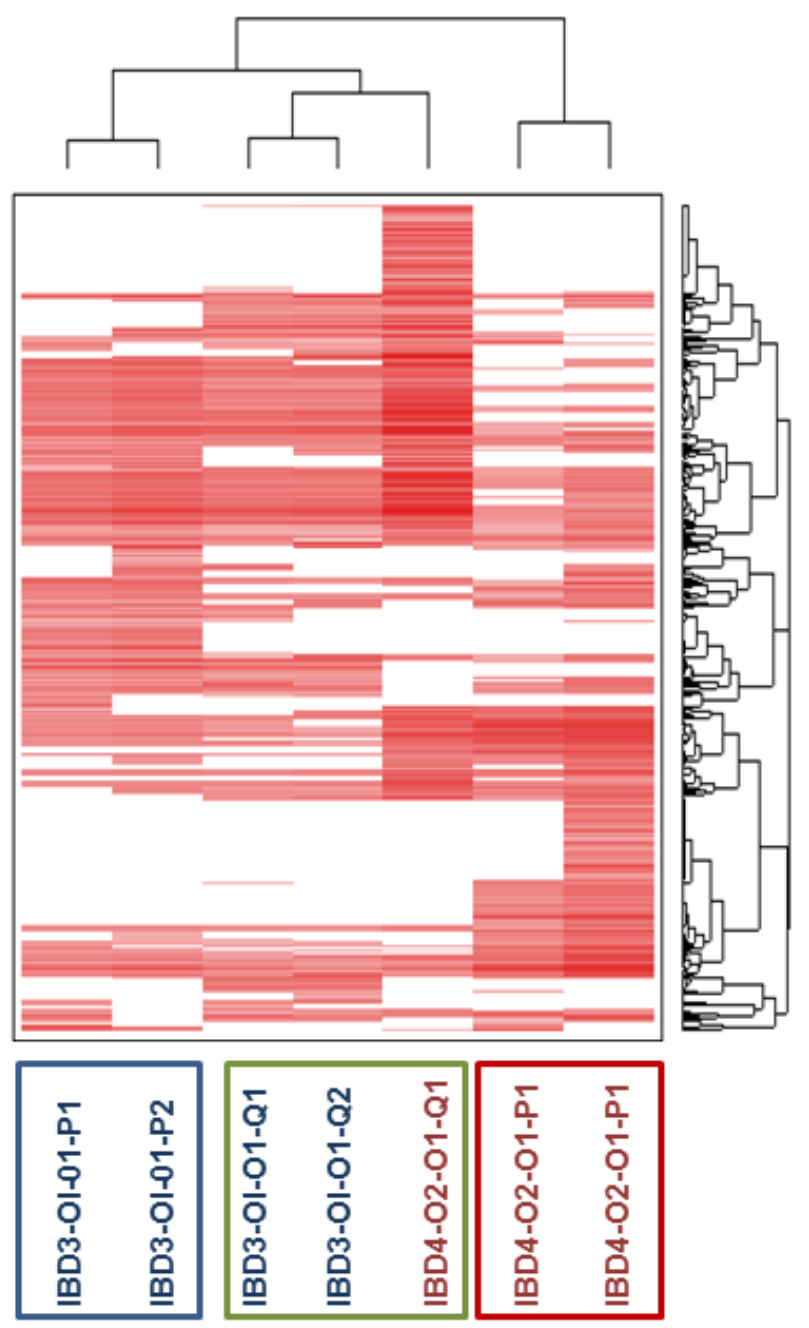

Figure 3: Cluster analysis of control samples: Cluster analysis was used to identify the effects of extraction methods on identification of microbial content and diversity in fecal samples. Samples clustered primarily by the extraction method followed by the origin of the sample. The intensity of the red indicates increasing amounts of the specific 16S Ribosomal target. Samples IBD3-01-01-P1 and IBD3-01-01-P2 clustered together while IBD301-01-Q1, IBD4-01-01-Q2 and IBD4-01-01-Q1 clustered together. Both phenol extractions of HM02 remained closely related. The effects of using $250 \mu \mathrm{g} v \mathrm{vs} 250 \mu \mathrm{l}$ was not significantly different.

the 66 species were present in the samples IBD3-01-01-Q1 and Q2, and IBD4-02-01-Q1. Only 3 of the 66 species were present in all 7 samples (Figure 4B). An abundance of Bacteroidetes species in fecal samples of humans has been previously reported $[15,60,61]$. Thus, the maximal representation of fecal microbiome represented by the hallmark bacterial species belonging to the phylum Bacteroidetes was detected using $250 \mu \mathrm{l}$ of stool sample extracted using the column method (Qiagen) following mechanical disruption $[54,62,63]$. This method was utilized in the rest of this study.

\section{Changes in the microbial diversity in IBD}

A marked decrease in the overall microbial diversity was observed in fecal samples from five IBD participants at the pre-diet modification time point compared to the negative controls. Forty-nine bacterial representative species belonging to 12 classes were decreased in $16 \mathrm{~S}$ gene levels while only 16 species belonging to 4 classes were present at higher levels in fecal samples from IBD patients (Figure 5). These results were consistent across $75 \%$ of the comparisons analyzed (6/8). There was a significant overlap of bacterial classes that were increased or decreased in the presence of IBD. The dominant classes with decreased abundance included some species of Clostridia (21\%), Bacilli (16\%) and Bacteroidetes (21\%). The classes represented in the increased abundance group include other species of Clostridia (50\%) and Gammaproteobacteria (30\%).

While the overall microbial diversity is decreased in IBD patients, some bacterial species belonging to Phylum Bacteroidetes were significantly increased in these pre-diet modification samples (Figure 6) [18,64]. Representative species, Bacteroides fragilis, was increased in all fecal samples from IBD patients, as evidences by increased fluorescence of the corresponding probe 


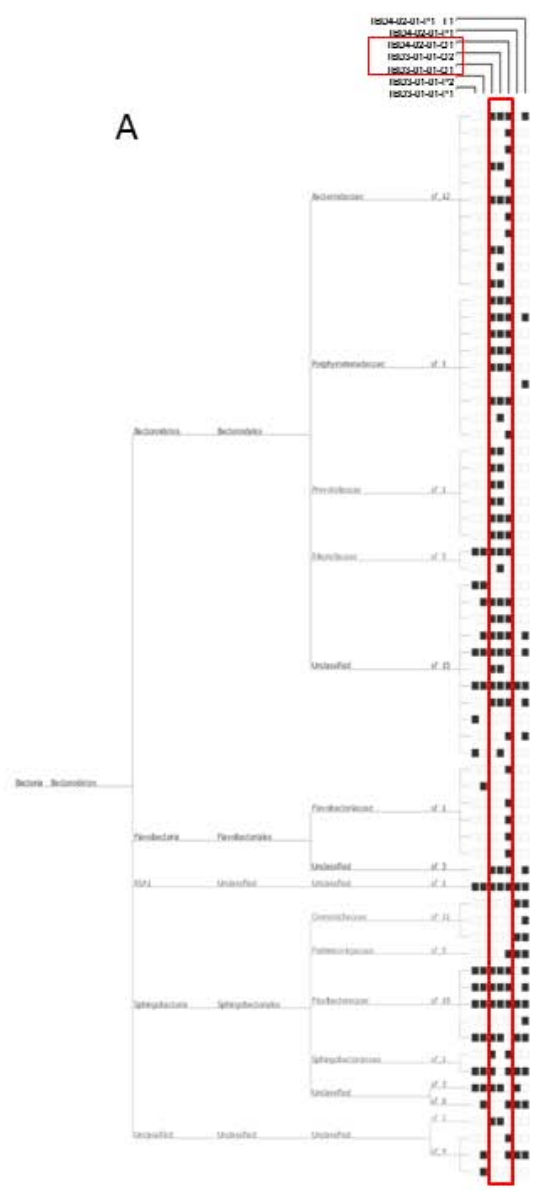

B

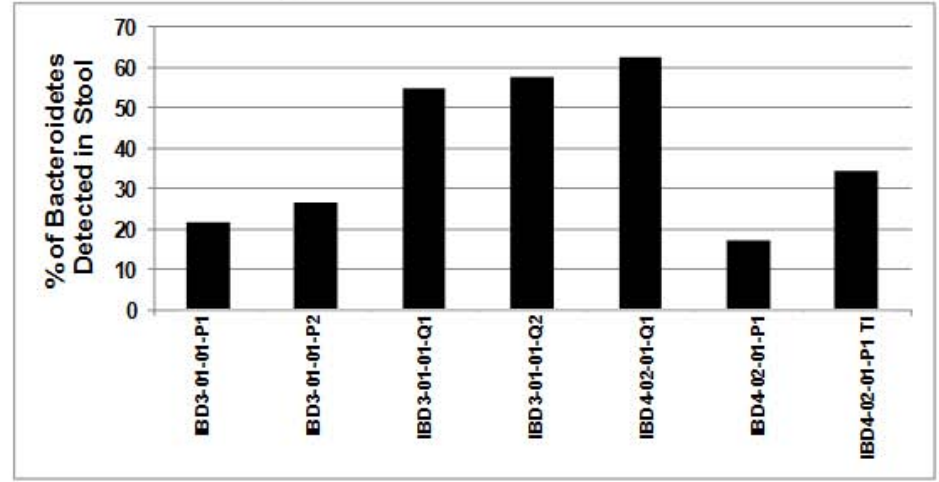

Figure 4

Figure 4: Detection of Bacteroidetes in fecal samples: Bacteroidetes is the most common and most abundant bacterial phylum present in human fecal samples. The maximal representation of this phylum was found using the Qiagen Fecal DNA extraction method Figure 4A and 4B $54-62 \%$ of the 66 species represented on the Phylochip Figure 4A. Three of 66 were present in all 7 sample types while 7 were present in 4 sample types. The best microbial representation was obtained using 250 $\mathrm{\mu l}$ Fecal and DNA extracted using the Qiagen Fecal DNA extraction method Figure 4B.

sets, and may indicate a shift in the composition of the microbiota from the controls. Blb. denitrificans, Ruminococcus torques, and other Bacteroides species, such a Bacteroides stercoris, were increased in abundance as indicated by higher fluorescence intensity of the specific probe set as well.

Clostridium lactatifermentans and other human colonic clones that have been previously identified in feces of healthy controls were decreased in the colons of IBD positive participants (Figure 7,9C). Clostridium leptum subgroup F. prausnitzii levels were increased in the fecal samples of IBD patients as has been previously described $[65,66]$. Decreased abundance of bacteria such as F.prausnitzii in colonic biopsies has been associated with an increase in symptoms of Crohn's ileitis. In this study, however, there was an increased representation of $F$. prausnitzii in the fecal samples, which could indicate a decreased proportion of the bacteria at the mucosal surface as previously reported due to increased shedding [67]. Some clones of $F$ prausnitzii, such as $C$. Leptum subgroup clone HuCB2 and p-5460-2Wb5, were decreased in abundance. The significance of these changes is unknown.

\section{Low residue diet (LRD) and the specific carbohydrate diet (SCD) affect fecal microbiome diversity}

A general increase in diversity was observed in fecal samples of participants on the SCD diet as compared to the LRD diet with a larger number of represented bacterial families showing changed abundance following diet modification (Figure 8,9). The SCD is enriched in simple carbohydrates. This was a longitudinal monitoring of participants who were serially sampled prior to initiation of diet modifications, following the initial SCD or LRD diet assignment, and after switching to the other diet option. The data is representative of individuals on the diet compared to their pre-diet samples. A period of wash-out was included between the two diet periods. The microbiome did not return to the composition of the pre-diet state following the washout period (data not shown). In fact, the change in the microbiome during the diet was retained during the period of washout.

Following the SCD, the microbial diversity increased to include 134 bacteria belonging to 32 different classes (Figure 8). The LRD diet was associated with a decreased diversity of the microbiome with 11 bacteria belonging to 3 families (Figure 


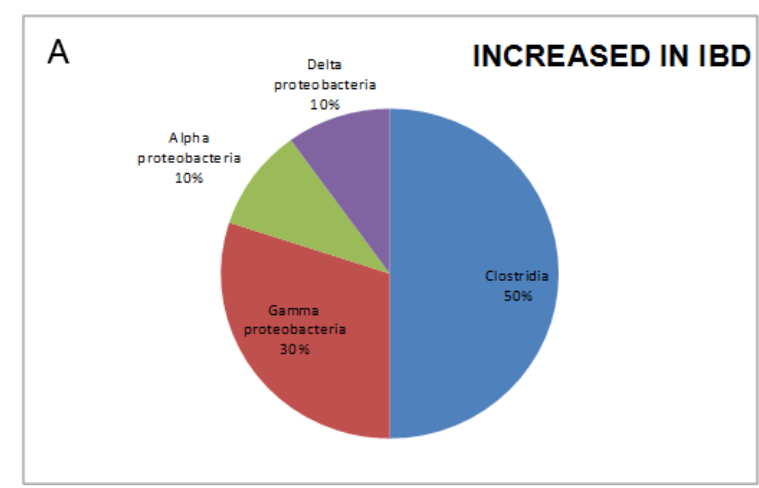

Figure 5

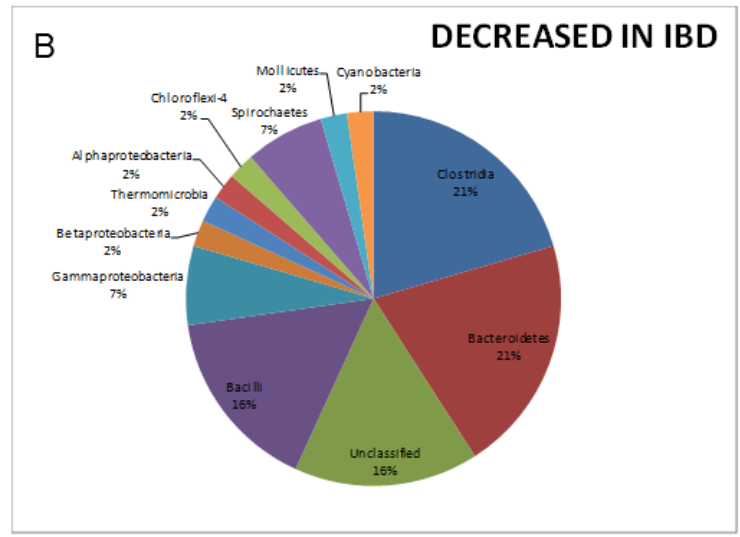

Figure 5: Stool samples obtained from patients with IBD showed a decrease in diversity of the fecal microbiome:

(A) While only 16 representative bacterial species belonging to 4 classes were seen at higher levels than controls, (B) 49 representative bacterial species belonging to 12 classes were decreased in abundance in greater than $75 \%$ or more of the IBD samples compared to normal controls.

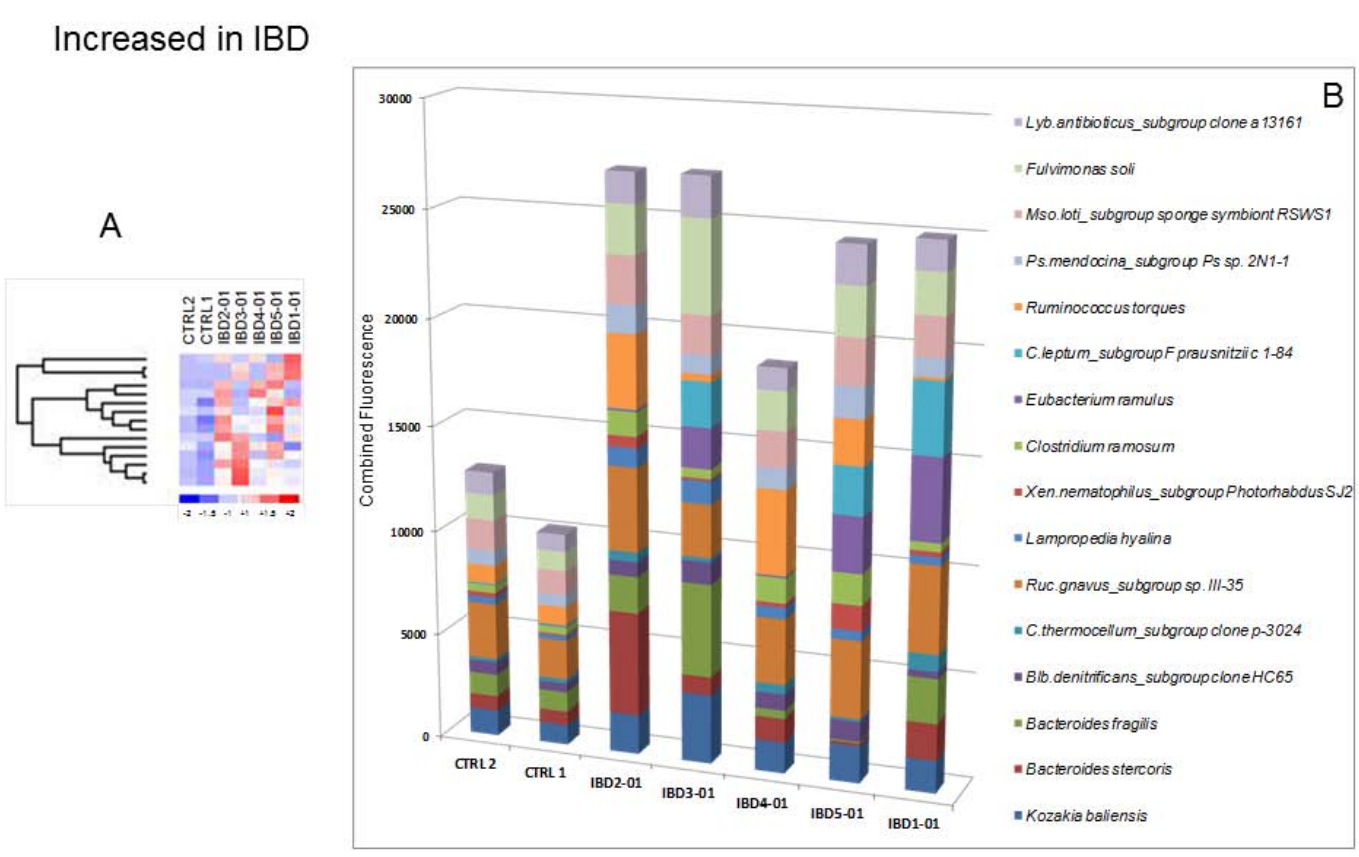

Figure 6: Bacterial species increased in expression in the fecal samples of patients with IBD: (A) Increase in expression of a limited number of bacterial species in the 5 IBD samples as compared to the non-IBD controls. The samples cluster according to clinical status (B) the most prominent species include B. fragilis and B. stercoris in 3 of the 5 samples obtained. The combined fluorescence was significantly higher in the 5 IBD samples as compared to the normal controls. 


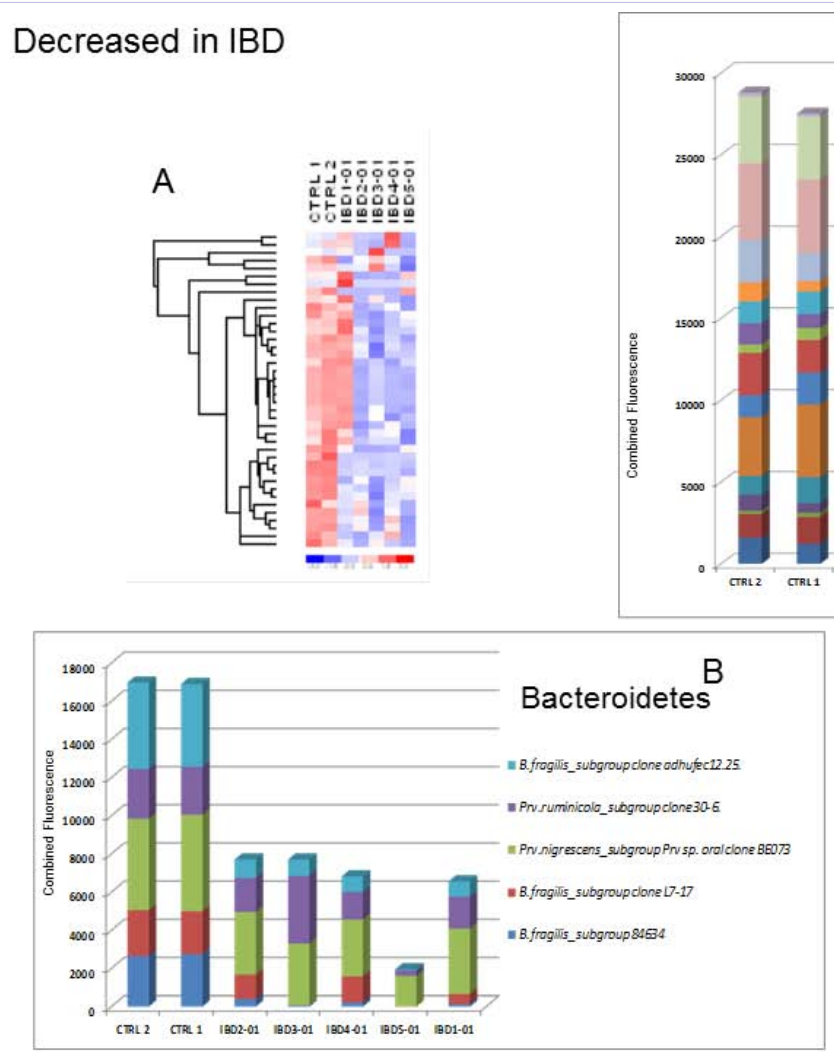

Figure 7

Figure 7: Bacterial species decreased in expression in the fecal samples of patients with IBD: (A) Hierarchical clustering demonstrated that the fecal samples clustered according to clinical state. 37 bacterial species were decreased in representation in the stool samples of IBD+ participants as compared to negative controls. The most prominent phyla included Bacteroidetes (B) and Clostridia (C).

9). The bacterial families overrepresented in the increase in SCD included over 20 species of the non-pathogenic clostridia family. Many of these species were decreased in the participants of the LRD diet. A shift in the representation of several bacteria of Clostridia spp was observed with the diet change. However, increased microbial diversity was not associated with any change in the clinical status.

\section{Discussion}

The pathogenesis of IBD is multifactorial and is a consequence of interplay between genetics, immune dysregulation and environmental factors. Within the last decade, advances in microbiome analysis have allowed a shift in focus to the importance of microbiota, its effects on intestinal homeostasis, and the development of IBD. An individual's characteristics such as age, body mass index, and gender do not completely determine the population and dominance of specific enterotypes but play a role in shaping it $[13,68]$. A recent study involving monozygotic twins and their respective biological mothers showed that families shared a very similar bacterial makeup [69]. Diet, body fat composition and a variety of infections modify the gut Microbiota $[7,70-74]$. These findings suggest that gut microbiome may be altered or shaped by other factors in addition to the genetic factors or the dynamics between exposure to specific viruses or bacteria and the host.

Intestinal epithelial permeability has been shown to be a determining factor in the development and progression of IBD and other inflammatory conditions. Disruption of the integrity of the tight junctions in the epithelial barrier, impaired mucin secretion and Paneth cell functions contribute to the increased permeability [75]. Epithelial barrier defects can be attributed to genetic susceptibility and gut inflammation $[76,77]$.The immunemodulators and biologic agents are being utilized to control gut inflammation. Another approach for controlling the intestinal inflammation may include reshaping the gut microbiota through diet interventions $[78,79]$. A change in the diet with potential inclusion of prebiotics and/or probiotics can alter the gut microbiota that is beneficial for human health. Our pilot study was focused on investigating the impact of two specific diets, in IBD patients, on the fecal microbiome in a controlled setting.

We first identified the effects of bacterial DNA extraction methodology on the representation of species complexity in the fecal microbiome as has been previously shown. The method of extraction played an important role in the types and number of species that were identified in the stool samples [54]. While there was no significant difference in the bacterial DNA yields using these two methods, the identification of Bacteroides Spp. in the fecal samples extracted by the column method was far greater than in the phenol chloroform method. Thus it is possible that the column method may be the choice method for use in fecal samples with similar downstream applications. While some 


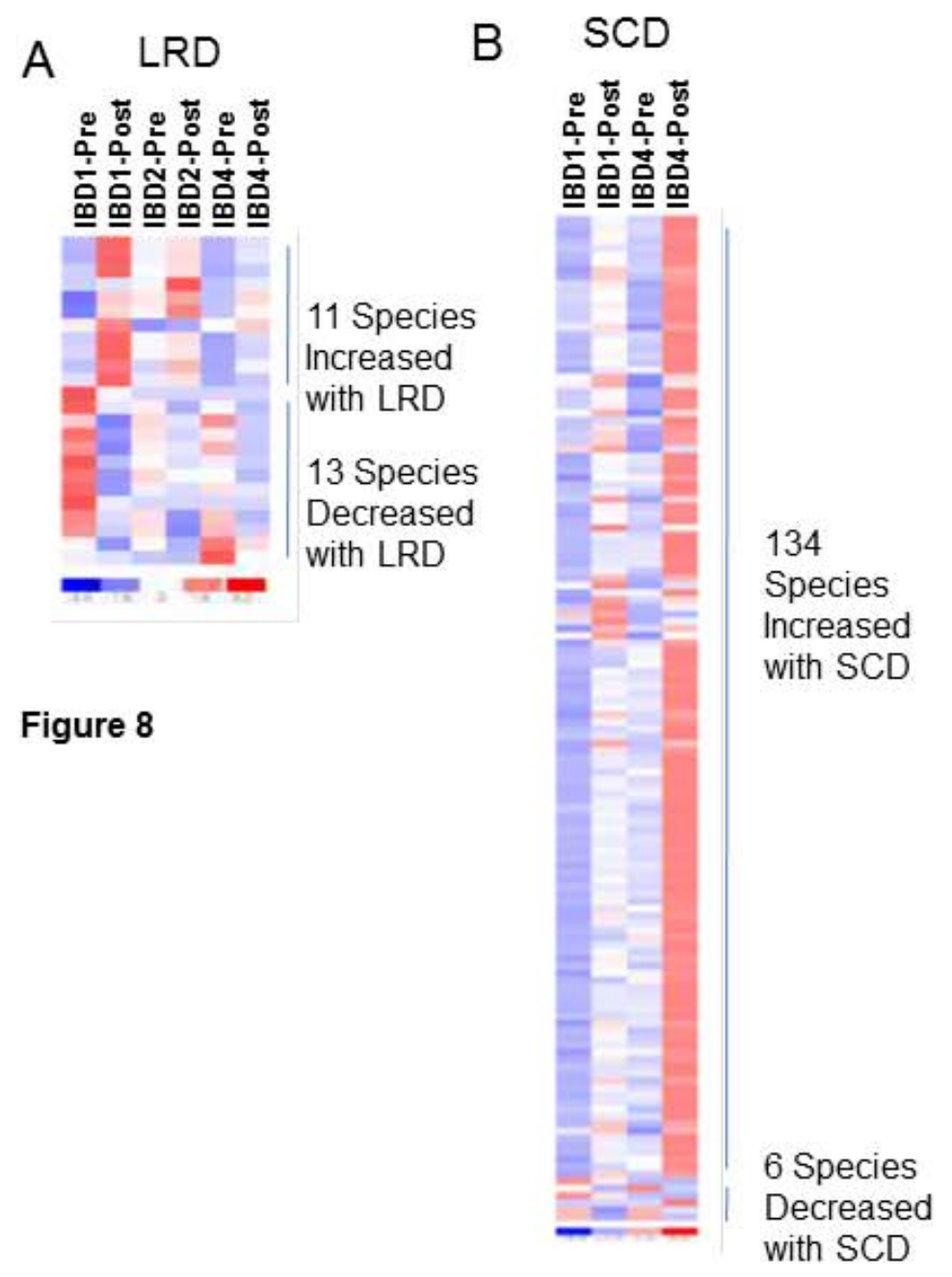

Figure 8: Increased in bacterial diversity is seen following SCD diet with no appreciable changes during LRD diet modification: Compared to the pre LRD diet stool samples from participants had an increase in levels of 11 species and a decrease in 13 species (A). In contrast the SCD diet change was associated with an increase in 134 species and a decrease on only 6 species (B). (Red: increase, Blue: decrease).

studies have found a wide variability in the representation of Bacteroides Spp, others have shown their presence consistently in human stool samples [63,80-82]. Furthermore, the use of DNA microarray technology provides a fast and convenient means to examine the alterations in the fecal microbiota on a large scale and provides a window into the health of the intestinal mucosa.

The role of microbiota in gut inflammation and IBD has been extensively studied [83-87]. The effects of Bacteroides fragilis, on the Th1 responses through the action of the bacterial-derived polysaccharide A (PSA) have been demonstrated. Furthermore, altered microbiota also play a role in activation of a Th17 response which is pro inflammatory, especially in IBD [83]. The global analysis of IBD associated dysbiosis has provided information on the complex interplay between microbiota, the innate and acquired immune system. The gap in knowledge is in the area of whether diet modifications can affect the microbiota in a positive manner and if this change is measurable. Our study has provided data to suggest that changes in the microbial diversity associated with IBD can be altered by dietary changes. While this study utilized a small " $n$ ", the longitudinal samples provided critical evidence of the effects of diet modification on the fecal microbiota. The level of gut inflammation was not characterized, however all participants had disease and it is assumed that gut inflammation played a role in the microbiome that was detected in the stool samples. At baseline, before diet implementation, overall microbial diversity was significantly decreased in IBD samples as compared to the healthy negative controls. IBD patients had more Bacteroides fragilis and a decreased abundance in Clostridium lactatifermentans, indicating a shift in the microbiota away from the composition of the microbial communities in the healthy controls. In terms of improving the microbial diversity that IBD patients lacked, the SCD diet proved to be more effective. Patients on the SCD diet 


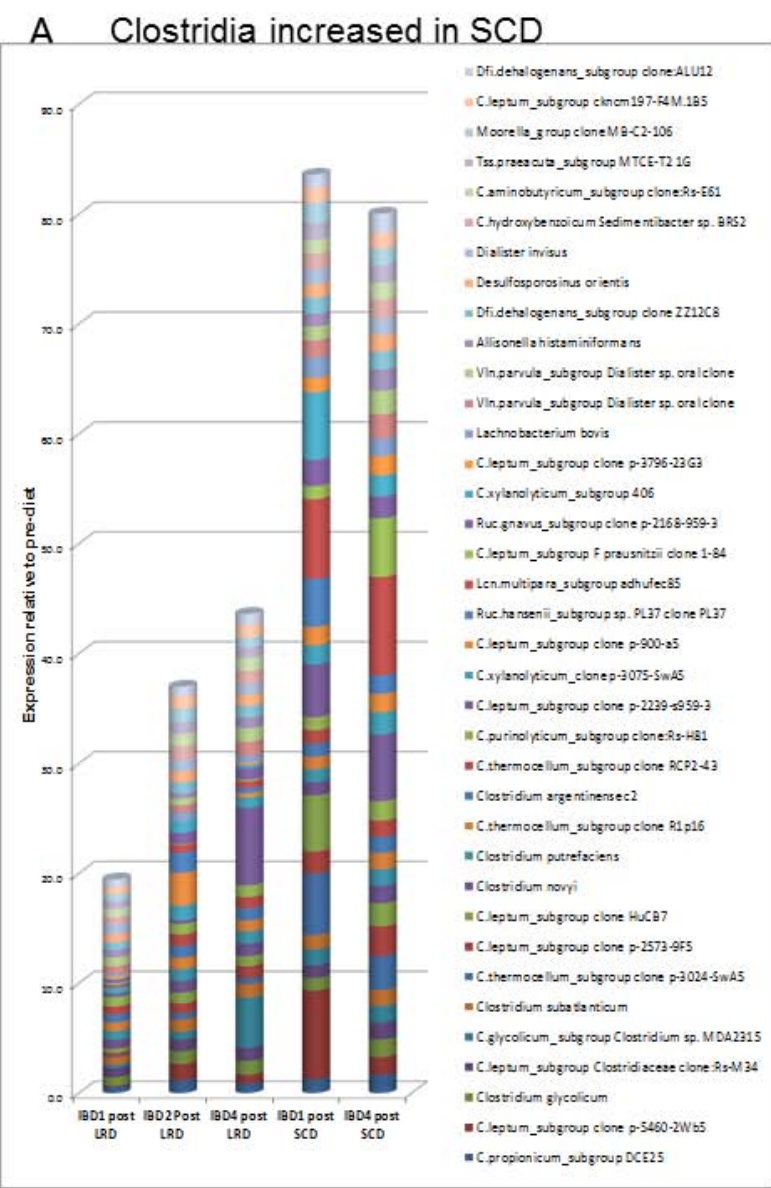

$\mathrm{B}$

Clostridia decreased in LRD

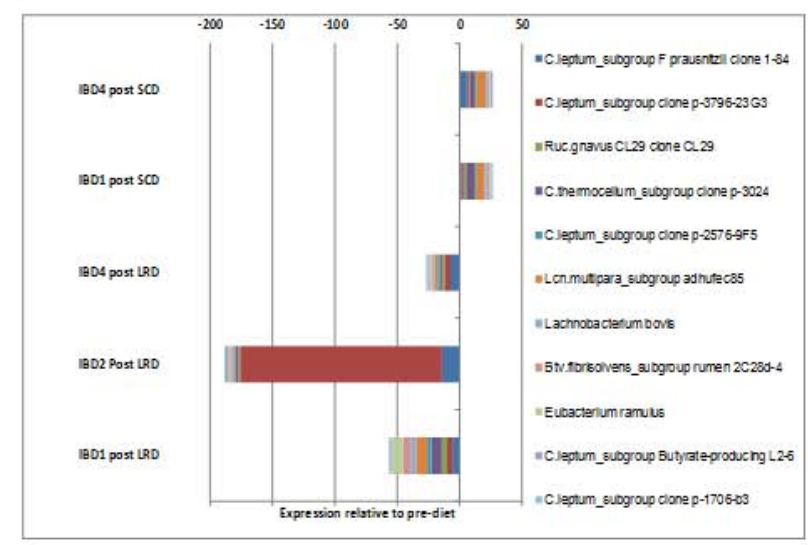

Figure 9: Effect of Diet modification on Clostridia species: The main component in the increased diversity (A) seen with SCD is made up of nonpathogenic clostridia species while many bacteria belonging to this class were decreased during the LRD diet (B).

had an increased abundance of some $C$. leptum species, which typically has been known to be a minor bacterial component in IBD patients $[66,67,88]$. Interestingly, the increase in microbial diversity with the SCD diet included an increased representation of $F$. prausnitzii, an anti-inflammatory commensal, in the stool samples [66]. More importantly, the gut Microbiome diversity was maintained and did not return to baseline composition during the washout periods. On the contrary, the LRD diet caused a drastic decrease in the Microbiome diversity. Due to the limited data, we were unable to show a significant clinical improvement with the increase in microbial diversity in IBD patients receiving the SCD diet.

Further investigations are warranted to explore specific diet regimens for clinical improvement in IBD patients and using the restructured gut microbial diversity as a correlate. Future studies of patient disease groups and controls will help delineate the host-microbe interactions in the gut that help maintain intestinal health.

\section{Acknowledgement}

We acknowledge Jimmy Pan, Steven Baumann and the UC Davis SOM Microarray Core Facility for technical support. We would like to thank the Research Department and the Nutrition Department at David Grant Medical Center, Travis Air Force Base, California. We would like to acknowledge Registered Dieticians, Major James Weinstein, Major Amanda Sager, and Major Holly Brewer for their assistance in this study

Sumathi Sankaran: Data analysis, manuscript preparation

Antonio Quiros: Study Design and implementation

Matthew Rolston: Experimental design and technical assistance

Irina Grishina: Data Analysis and manuscript preparation

Jay L: Data Analysis

Anne Fenton: Manuscript preparation

Todd Z. De Santis: Data Analysis

Anne Thai: Manuscript preparation

Gary L Andersen: Study Design and implementation

Peggy Papathakis: Study Design and implementation

Raquel Nieves: Study Design and implementation 
Thomas Prindiville: Study Design and implementation

Satya Dandekar: Study Design and implementation

\section{Funding}

This study was supported by grants from the National Institute of Health (NIH) R01 (DK61297, AI43274). Dr. Sankaran is supported by a Building Interdisciplinary Research Careers in Women's Health award (K12 HD051958) funded by the NICHD, ORWH, and the NIA.

\section{References}

1. Yatsunenko T, Rey FE, Manary MJ, et al. Human gut microbiome viewed across age and geography. Nature. 2012; 486(7402): 222-227.

2. Structure, function and diversity of the healthy human microbiome. Nature. 2012; 486(7402): 207-214.

3. Ghosh S, Dai C, Brown K, et al. Colonic microbiota alters host susceptibility to infectious colitis by modulating inflammation, redox status, and ion transporter gene expression. Am J Physiol Gastrointest Liver. Physiol. 2011; 301(1): 39-49.

4. Dave M, Higgins PD, Middha S, et al. The human gut microbiome: current knowledge, challenges, and future directions. Transl Res. 2012; $160(4): 246-57$.

5. Lawson RD, Coyle WJ. The noncolonic microbiome: does it really matter?Curr Gastroenterol Rep. 2010; 12(4): 259-262.

6. Schwiertz A, Taras D, Schafer K, et al. Microbiota and SCFA in lean and overweight healthy subjects. Obesity (Silver Spring). 2010; 18(1): 190-195

7. Turnbaugh PJ, Hamady M, Yatsunenko T, et al. A core gut microbiome in obese and lean twins. Nature. 2009; 457(7228): 480-484.

8. Li M, Wang B, Zhang M, et al. Symbiotic gut microbes modulate human metabolic phenotypes. Proc Natl Acad Sci USA 2008; 105(6): 21172122.

9. Sobhani I, Tap J, Roudot-Thoraval F, et al. Microbial dysbiosis in colorectal cancer (CRC) patients. PLoS One. 2011; 6(1): e16393.

10. Balter M. Taking stock of the human microbiome and disease. Science. 2012; 336(6086): 1246-1247.

11.Zimmermann MB, Chassard C, Rohner F, et al. The effects of iron fortification on the gut microbiota in African children: a randomized controlled trial in Cote d'Ivoire. Am J Clin Nutr2010; 92(6): 14061415.

12. Walker AW, Ince J, Duncan SH, et al. Dominant and diet-responsive groups of bacteria within the human colonic microbiota. ISME J. 2011; 5(2): 220-230.

13. Arumugam M, Raes J, Pelletier E, et al. Enterotypes of the human gut microbiome. Nature. 2011; 473 (7346): 174-180.

14. Mc Donald JA, Schroeter K, Fuentes S, et al. Evaluation of microbial community reproducibility, stability and composition in a human distal gut chemostat model. J microbiol methods. 2013; 95(2): 167174.

15. Gill SR, Pop M, Deboy RT et al. Metagenomic analysis of the human distal gut microbiome. Science. 2006; 312(5778): 1355-1359.

16. Contreras M, Costello EK, Hidalgo G, et al. The bacterial microbiota in the oral mucosa of rural Amerindians. Microbiology. 2010; 156(11): 3282-3287.
17. Magrone T, Jirillo E. The interaction between gut microbiota and agerelated changes in immune function and inflammation. Immunity \& ageing. 2013; 10(1): 31.

18. Seksik P, Rigottier-Gois L, Gramet G, et al. Alterations of the dominant faecal bacterial groups in patients with Crohn's disease of the colon. Gut. 2003; 52(2): 237-242.

19. Marteau P, Lepage P, Mangin I,et al. Review article: gut flora and inflammatory bowel disease. Aliment Pharmacol Ther 2004; 20(4): 18-23.

20.Walker AW, Sanderson JD, Churcher C, et al. High-throughput clone library analysis of the mucosa-associated microbiota reveals dysbiosis and differences between inflamed and non-inflamed regions of the intestine in inflammatory bowel disease. BMC Microbiol. 2011; 11:7.

21. Kang S, Denman SE, Morrison M, et al. Dysbiosis of fecal microbiota in Crohn's disease patients as revealed by a custom phylogenetic microarray. Inflamm Bowel Dis. 2010; 16(12): 2034-2042.

22. Yildirim S, Yeoman CJ, Sipos M, et al. Characterization of the fecal microbiome from non-human wild primates reveals species specific microbial communities. PLoS One. 2010; 5(11): e13963.

23. Pepper JW, Rosenfeld S. The emerging medical ecology of the human gut microbiome. Trends Ecol Evol. 2012; 27(7): 381-384.

24. Green Johnson JM. Immunological responses to gut bacteria. J AOAC Int. 2012; 95(1): 35-49.

25. Ashida H, Ogawa M, Kim M, et al. Bacteria and host interactions in the gut epithelial barrier. Nat Chem Biol. 2011; 8(1): 36-45.

26. Paliy 0, Agans R. Application of phylogenetic microarrays to interrogation of human microbiota. FEMS Microbiol Ecol. 2012; 79(1): 2-11.

27. Cox MJ, Huang YJ, Fujimura KE, et al. Lactobacillus casei abundance is associated with profound shifts in the infant gut microbiome. PLoS One. 2010; 5(1): e8745.

28. Codling C, O Mahony L, Shanahan F, et al. A molecular analysis of fecal and mucosal bacterial communities in irritable bowel syndrome. Dig Dis Sci. 2010; 55(2): 392-397.

29. McKenna P, Hoffmann C, Minkah N, et al. The macaque gut microbiome in health, lentiviral infection, and chronic enterocolitis. PLoS Pathog. 2008; 4(2): e20.

30.0'Keefe SJ. Nutrition and colonic health: the critical role of the microbiota. Curr Opin Gastroenterol 2008;24(1):51-58.

31. Godoy-Vitorino F, Goldfarb KC, Karaoz U, et al. Comparative analyses of foregut and hindgut bacterial communities in hoatzins and cows. ISME J. 2012; 6(3): 531-541.

32. Radosevich JL, Wilson WJ, Shinn JH, et al. Development of a highvolume aerosol collection system for the identification of air-borne micro-organisms. Lett Appl Microbiol. 2002; 34(3):162-167.

33. Brodie EL, DeSantis TZ, Parker JP, et al. Urban aerosols harbor diverse and dynamic bacterial populations. Proc Natl Acad Sci USA. 2007; 104(1): 299-304.

34. Brodie EL, Desantis TZ, Joyner DC, et al. Application of a high-density oligonucleotide microarray approach to study bacterial population dynamics during uranium reduction and reoxidation. Appl Environ Microbiol. 2006; 72(9): 6288-6298.

35. Finegold SM, Attebery HR, Sutter VL. Effect of diet on human fecal flora: comparison of Japanese and American diets. Am J Clin Nutr. 1974; 27(12): 1456-1469. 
36. Hill MJ: Diet and cancer. Eur J Cancer Prev. 1995; 4(6): 443-444.

37.Wu GD, Chen J, Hoffmann C, et al. Linking long-term dietary patterns with gut microbial enterotypes. Science. 2011; 334(6052): 105-108.

38. Omura K, Hirano K, Kanehira E, et al. Small amount of low-residue diet with parenteral nutrition can prevent decreases in intestinal mucosal integrity. Ann Surg. 2000; 231(1): 112-118.

39. Koga $\mathrm{H}$, Iida M, Aoyagi K, et al. [Long-term efficacy of low residue diet for the maintenance of remission in patients with Crohn's disease]. Nihon Shokakibyo Gakkai Zasshi. 1993; 90(11): 2882-2888.

40. Chapkin RS, Kamen BA, Callaway ES, et al. Use of a novel genetic mouse model to investigate the role of folate in colitis-associated colon cancer. J Nutr Biochem. 2009; 20(8): 649-655.

41. Mahmud N, Weir DG. The urban diet and Crohn's disease: is there a relationship?Eur J Gastroenterol Hepatol. 2001; 13(2): 93-95.

42. Berti C, Riso P, Monti LD, et al. In vitro starch digestibility and in vivo glucose response of gluten-free foods and their gluten counterparts. Eur J Nutr. 2004; 43(4): 198-204.

43. Haas SV, Haas MP. The treatment of celiac disease with the specific carbohydrate diet; report on 191 additional cases. Am J Gastroenterol. 1955; 23(4): 344-360.

44. Scott KP, Gratz SW, Sheridan PO, et al. The influence of diet on the gut microbiota. Pharmacological research: the official journal of the Italian Pharmacological Society. 2013; 69(1): 52-60.

45. Flint HJ, Scott KP, Duncan SH, et al. Microbial degradation of complex carbohydrates in the gut. Gut microbes. 2012; 3(4): 289-306.

46. Lionetti P, Callegari ML, Ferrari S, et al. Enteral nutrition and microflora in pediatric Crohn's disease. JPEN J Parenter Enteral Nutr. 2005; 29(4 Suppl): S173-175.

47. Matsui T, Ueki M, Yamada M, et al. Indications and options of nutritional treatment for Crohn's disease. A comparison of elemental and polymeric diets. J Gastroenterol. 1995; 30 Suppl 8: 95-97.

48. Russell RI: Review article: dietary and nutritional management of Crohn's disease. Aliment Pharmacol Ther. 1991; 5(3): 211-226.

49. Aoki Y, Ohta M, Sakamoto Y, et al. Application of solid low residue diets consisting mainly of elemental diet in colorectal diseases. Nihon Geka Hokan. 1986; 55(2): 358-366.

50. Brown AC, Roy M. Does evidence exist to include dietary therapy in the treatment of Crohn's disease?Expert review of gastroenterology \& hepatology. 2010; 4(2): 191-215.

51. Brown AC, Valiere A. Probiotics and medical nutrition therapy. Nutrition in clinical care : an official publication of Tufts University. 2004; 7(2): 56-68.

52. D'Aversa F, Tortora A, Ianiro G, et al. Gut microbiota and metabolic syndrome. Internal and emergency medicine. 2013; 8 (Suppl 1): S1115.

53.Zoetendal EG, de Vos WM. Effect of diet on the intestinal microbiota and its activity. Curr Opin Gastroenterol. 2014; 30(2): 189-195.

54. P OC, Aguirre de Carcer D, Jones M, et al. The effects from DNA extraction methods on the evaluation of microbial diversity associated with human colonic tissue. Microb Ecol. 2011; 61(2): 353-362.

55. Kim M, Morrison M, Yu Z. Evaluation of different partial 16S rRNA gene sequence regions for phylogenetic analysis of microbiomes. Journal of microbiological methods. 2011; 84(1): 81-87.
56. Yergeau E, Schoondermark-Stolk SA, Brodie EL, et al. Environmental microarray analyses of Antarctic soil microbial communities. ISME J. 2009; 3(3): 340-351.

57.Schatz MC, Phillippy AM, Gajer P, et al. Integrated microbial survey analysis of prokaryotic communities for the PhyloChip microarray. Appl Environ Microbiol. 2010; 76(16): 5636-5638.

58. Li C, Hung Wong W. Model-based analysis of oligonucleotide arrays: model validation, design issues and standard error application. Genome Biol. 2001; 2(8):RESEARCH0032.

59. Shedden K, Chen W, Kuick R, et al. Comparison of seven methods for producing Affymetrix expression scores based on False Discovery Rates in disease profiling data. BMC Bioinformatics. 2005; 6:26.

60. Eckburg PB, Bik EM, Bernstein CN, et al. Diversity of the human intestinal microbial flora. Science. 2005; 308(5728): 1635-1638.

61. Dethlefsen L, Huse S, Sogin ML, et al. The pervasive effects of an antibiotic on the human gut microbiota, as revealed by deep $16 \mathrm{~S}$ rRNA sequencing. PLoS Biol. 2008; 6(11): e280.

62. Human Microbiome Project C. Structure, function and diversity of the healthy human microbiome. Nature. 2012; 486(7402): 207-214.

63. Goll J, Thiagarajan M, Abubucker S, et al. A case study for large-scale human microbiome analysis using JCVI's metagenomics reports (METAREP). PLoS One. 2012; 7(6): e29044.

64. Andoh A, Tsujikawa T, Sasaki M, et al. Faecal microbiota profile of Crohn's disease determined by terminal restriction fragment length polymorphism analysis. Aliment Pharmacol Ther. 2009; 29(1): 75-82.

65. Martinez C, Antolin M, Santos J, et al. Unstable composition of the fecal microbiota in ulcerative colitis during clinical remission. Am J Gastroenterol. 2008; 103(3): 643-648.

66. Flanagan P, Campbell BJ, Rhodes JM: Bacteria in the pathogenesis of inflammatory bowel disease. Biochem Soc Trans. 2011; 39(4): 1067 1072.

67.Willing BP, Dicksved J, Halfvarson J, et al. A pyrosequencing study in twins shows that gastrointestinal microbial profiles vary with inflammatory bowel disease phenotypes. Gastroenterology. 2010; 139(6): 1844-1854.e1.

68. Siezen RJ, Kleerebezem M. The human gut microbiome: are we our enterotypes?Microb Biotechnol2011;4(5):550-553.

69. Reyes A, Haynes M, Hanson N, et al. Viruses in the faecal microbiota of monozygotic twins and their mothers. Nature. 2010; 466(7304): 334-338.

70. Gophna U. Microbiology. The guts of dietary habits. Science. 2011; 334(6052): 45-46.

71. De Cruz P, Prideaux L, Wagner J, et al. Characterization of the gastrointestinal microbiota in health and inflammatory bowel disease. Inflamm Bowel Dis. 2012; 18(2): 372-390.

72. Klewicka E, Cukrowska B, Libudzisz Z, et al. Changes in gut microbiota in children with atopic dermatitis administered the bacteria Lactobacillus casei DN--114001. Pol J Microbiol. 2011; 60(4): 329333.

73. Turnbaugh PJ, Ridaura VK, Faith JJ, et al. The effect of diet on the human gut microbiome: a metagenomic analysis in humanized gnotobiotic mice. Sci Transl Med. 2009; 1(6): 6ra14.

74. Flier JS, Mekalanos JJ. Gut check: testing a role for the intestinal microbiome in human obesity. Sci Transl Med. 2009; 1(6): 6ps7. 
75. Hollander D. Crohn's disease--a permeability disorder of the tight junction?Gut. 1988; 29(12): 1621-1624.

76. Secondulfo M, de Magistris L, Fiandra R. Intestinal permeability in Crohn's disease patients and their first degree relatives. Dig Liver Dis. 2001; 33(8): 680-685.

77. Murphy MS, Eastham EJ, Nelson R, et al. Intestinal permeability in Crohn's disease. Arch Dis Child. 1989; 64(3): 321-325.

78. Paturi G, Mandimika T, Butts CA, et al. Influence of dietary blueberry and broccoli on cecal microbiota activity and colon morphology in mdr1a(-/-) mice, a model of inflammatory bowel diseases. Nutrition. 2012; 28(3): 324-330.

79. Haller D. Nutrigenomics and IBD: the intestinal microbiota at the crossroad between inflammation and metabolism. J Clin Gastroenterol. 2010; 44(Suppl 1): S6-9.

80.Zubrzycki L, Spaulding EH. Studies on the Stability of the Normal Human Fecal Flora. Journal of bacteriology. 1962; 83(5): 968-974.

81. San Joaquin VH, Griffis JC, Lee C, et al. Association of Bacteroides fragilis with childhood diarrhea. Scandinavian journal of infectious diseases. 1995; 27(3):211-215.

82. Yuan J, Zeng B, Niu R, et al. The development and stability of the genus
Bacteriodes from human gut microbiota in HFA mice model. Current microbiology. 2011; 62(4): 1107-1112.

83. Ivanov II, Frutos Rde L, Manel N, et al. Specific microbiota direct the differentiation of IL-17-producing T-helper cells in the mucosa of the small intestine. Cell host \& microbe. 2008; 4(4): 337-349.

84. Alexander KL, Targan SR, Elson CO, $3^{\text {rd }}$. Microbiota activation and regulation of innate and adaptive immunity. Immunological reviews. 2014; 260(1): 206-220.

85. Comito D, Cascio A, Romano C. Microbiota biodiversity in inflammatory bowel disease. Italian journal of pediatrics. 2014; 40:32.

86. Ray K. IBD. Understanding gut microbiota in new-onset Crohn's disease. Nature reviews Gastroenterology \& hepatology. 2014; 11(5): 268.

87. Goldsmith JR, Sartor RB. The role of diet on intestinal microbiota metabolism: downstream impacts on host immune function and health, and therapeutic implications. J Gastroenterol. 2014; 49(5): 785-798.

88. Flanagan JL, Brodie EL, Weng L, et al. Loss of bacterial diversity during antibiotic treatment of intubated patients colonized with Pseudomonas aeruginosa. J Clin Microbiol. 2007; 45(6): 1954-1962. 